\title{
Construcción y aplicación de una herramienta metodológica para priorizar ecosistemas estratégicos: caso humedales del municipio Medio Atrato, Chocó, Colombia
}

\author{
Construction and application of a methodologic tool to prioritize strategic \\ ecosystems: case wetlands of municipality Medio Atrato, Chocó, Colombia
}

\author{
Giovanny Ramírez ${ }^{1}$, Teófilo Cuesta ${ }^{2}$, William Klinger ${ }^{3}$

\section{RESUMEN}

La construcción y aplicación de una herramienta metodológica para priorizar ecosistemas estratégicos, fue aplicada como mecanismo de selección de una muestra para estudiar un ecosistema muy amplio. Su aplicación en el complejo de humedales del Medio Atrato, Chocó, se realizó con el objetivo de establecer el nivel de prioridad de las ciénagas en estudio. La primera fase del proyecto consistió en determinar el número, ubicación, extensión, importancia socioeconómica y el grado de vulnerabilidad de las ciénagas; la segunda consistió en priorizar los humedales, teniendo en cuenta un peso relativo para cada uno de los criterios, así: extensión de la ciénaga, 20\% del peso total; ubicación, 35\%; importancia socioeconómica 45\%. Entre los resultados se destaca el hecho de que el ecosistema está constituido de 47 ciénagas; adicionalmente, se reportan tres actividades económicas que influyen en el uso de las mismas. Con base en la ponderación de los tres criterios para establecer el grado de vulnerabilidad y priorización de las ciénagas, se determinó que 13 de las 47 ciénagas (27,7\%) se encuentran en vulnerabilidad Alta (A), 27, $(57,4 \%)$ se encuentran en vulnerabilidad Media (M) y siete (7) (14,8\%) reportan vulnerabilidad Baja $(B)$.

Palabras clave: Ecosistemas estratégicos; Ciénagas; Herramienta metodológica; Priorización.

\begin{abstract}
The construction and application of a methodologic tool to prioritize strategic ecosystems, were applied as mechanism of selection of a sample to study a very ample ecosystem. Its application in the complex of moist soils of the Medio Atrato, Hit, this was realised with the aim of establishing the level of priority of bogs in study. First stage of the project consisted of determining the number, location, extension, socioeconomic importance and the degree of vulnerability of bogs; second it consisted of prioritizing moist soils, considering a relative weight for each one of the criteria, thus: extension of the bog a $20 \%$ of the gross weight, the location a $35 \%$ and the socioeconomic importance a $45 \%$. Between the results the fact stands out that the constituted ecosystem this of 47 bogs; additionally, three economic activities are reported that influence the use of the same. With base in the consideration of the three criteria to establish the degree of vulnerability and priorizacion of bogs, one determined that 13 of 47 bogs (27.7\%) are in High vulnerability (A), 27 bogs (57.4\%) are in Average vulnerability $(M)$ and seven (7) bogs (14.8\%) report Low vulnerability (B).
\end{abstract}

1. Biólogo, Magíster en Ciencias Biológicas (C); Investigador principal Componente Ecosistémico, Instituto de Investigaciones Ambientales del Pacífico (IIAP), Quibdó, Colombia. e-mail:gramirezm3@ hotmail.com

2. Ingeniero Forestal, Msc. Profesor Titular de la Universidad Distrital Francisco José De Caldas, Facultad de Medio Ambiente y Recursos Naturales, Grupo de Investigación PROPOBOS y Director del Instituto de Investigaciones Ambientales del Pacífico (IIAP), Quibdó, Colombia.

e-mail:wklinger@iiap.org.co

3. Ingeniero Agrónomo, Especialista en Gestión Ambiental, Universidad Tecnológica del Chocó, Presidente Fundación Agricultura con Enfoque Humano (FAEH), Quibdó, Colombia. e-mail: tcuesta@earth.ac.cr tcuesta@iiap.org.co Recibido: Junio 1, 2009 Aceptado: Junio 17, 2009 
Herramienta metodológica para priorizar ecosistemas estratégicos. G Ramírez et al.

Keywords: Strategic ecosystems; Moist soils; Methodologic tool; Priorization.

\section{INTRODUCCIÓN}

Seleccionar un área significativa de muestreo para caracterizar ecosistemas de gran tamaño resulta ser un reto para los investigadores, porque se deben tener en cuenta muchas variables a la hora elegir. De ahí que construir herramientas que faciliten este proceso sea el paso previo a la realización de caracterizaciones ambientales y estudios específicos dentro del área que permitan tomar decisiones relacionadas con el manejo de los recursos.

En este caso el río Atrato cuenta con un área de tierras bajas anegadizas, con grandes pantanos, caños y ciénagas reguladas por el nivel de este río, las cuales se encuentran en el medio y bajo Atrato. En el sector correspondiente al municipio del Medio Atrato, se encontró un complejo de alrededor de 47 ciénagas de distintas extensiones ubicadas en el área que corresponde a este municipio, siendo las más importantes: La Quesada, La Honda, La Grande, Casabe entre otras. Estas ciénagas hacen parte de un complejo ubicado en la parte media del río Atrato; permanecen inundadas la mayor parte del año, algunas de estas se encuentran aisladas, pero en su mayoría forman una red y se comunican entre si; muchas mantiene un nivel constante de agua, mientras que en otras varía en épocas de cese de lluvias y a medida que baja el nivel del río. Estos ecosistemas son de vital importancia para las comunidades locales, juegan un papel primordial a nivel cultural, porque los recursos que ofrecen constituyen la base de la economía de las comunidades asentadas en las riveras de los ríos; además sustentan una importante diversidad biológica y en muchos casos constituyen hábitats críticos para especies seriamente amenazadas (cativo), y por su alta productividad, pueden albergar poblaciones muy numerosas. Sin embargo, actualmente estos humedales están siendo amenazados por diferentes acciones antrópicas, como la deforestación y la minería, actividades que se realizan en las ciénagas o cerca de ellas.

Con este estudio se pretendió determinar el número y ubicación de las ciénagas así como la priorización de una de ellas, en la cual se realizara una caracterización ambiental, como aproximación al manejo sostenible de los complejos de humedales del Chocó, Colombia. La herramienta diseñada toma de cada una su extensión, ubicación, usos e importancia cultural, como criterios que ponderados permitieron obtener un grado de vulnerabilidad con el que se priorizó la ciénaga grande de la comunidad de Beté, en la cual se realizó una caracterización ambiental que describe y analiza biofísicamente el ecosistema desde varios componentes.

\section{METODOLOGÍA}

Número, ubicación, extensión, importancia socioeconómica y grado de vulnerabilidad de las ciénagas. En primer lugar, para determinar la cantidad y la ubicación geográfica precisa de los humedales del territorio en consideración se llevó a cabo un recorrido por las ciénagas que no se encontraban georeferenciadas y de las cuales se posee muy poca información. Este recorrido permitió localizare incluir las ciénagas en cartografía, las cuales se identificaron por los pobladores a través de talleres, en donde se elaboró a mano alzada un mapa de la zona donde los pobladores identificaron las ciénagas de su entorno con nombre, ubicación general en ríos o quebradas, así como su uso actual.

Para determinar la extensión de los diferentes humedales se utilizó un Sistema de Información Geográfica (SIG) asícomo rutas, períodos de accesibilidad proporcionado por los pobladores en los diferentes talleres y corroborado en campo. El establecimiento de la importancia socioeconómica y el grado de vulnerabilidad de cada uno de los humedales, se llevó a cabo con la ayuda de entrevistas a líderes comunitarios, quienes con base en su vivencia permanente aportaron elementos complementarios para el análisis in situ del equipo técnico; entre dichos elementos aportados por las comunidades se encuentran: usos, frecuencias de utilización de los recursos y técnicas de aprovechamiento, entre otros.

Priorización de los humedales. En la priorización se utilizó información generada a partir de observaciones in situ del equipo técnico y el desarrollo de entrevistas a personas locales; con base en esta información se ponderaron los humedales, teniendo en cuenta los siguientes criterios: extensión, ubicación e importancia socioeconómica para determinar el grado de vulnerabilidad. En el marco de dicha ponderación de criterios, se dio un valor para cada humedal en una escala de 1 a 5; al final se organizaron los ecosistemas en orden de prioridad, de acuerdo con el puntaje total obtenido.

Extensión. Se refiere al área cubierta por el ecosistema. Para establecer la calificación correspondiente a cada ciénaga en este aspecto se utilizaran los rangos mostrados en la Tabla 1.

Ubicación. Se refiere al orden de la ciénaga, siendo las ciénagas primarias o de tipo 1 aquellas que están conectadas directamente con el río; las ciénagas secundarias o de tipo 2, aquellas que están conectadas directa o indirectamente con el río a través de las ciénagas primarias y por último las ciénagas terciarias o de tipo 3 aquellas conectadas al río directa o indirectamente a través de las ciénagas secundarias o por medio del caño de una ciénaga secundaria. Las ciénagas de tipo 1 se calificaron con 5 puntos, las de tipo 2 con 3 y las 
Tabla 1

Rangos de calificación del criterio extensión de las ciénagas

\begin{tabular}{cc}
\hline Rango de extensión (ha) & Valor de calificación \\
\hline $0-80$ & 1 \\
$80-160$ & 2 \\
$160-240$ & 3 \\
$240-320$ & 4 \\
$320-400$ & 5 \\
\hline
\end{tabular}

de tipo 3 obtuvieron una calificación de 1 punto.

Importancia socioeconómica. Se refiere a los usos directos e indirectos que hace la comunidad de dichos ecosistemas. La calificación para cada ciénaga se determinó teniendo en cuenta el número de actividades productivas realizadas en las ciénagas y el grado de impacto que generan las mismas; los valores se obtiene como se muestra en la Tabla 2.

Grado de vulnerabilidad. Este criterio se refiere al grado de afectación ambiental y resulta de la ponderación de los criterios de extensión, importancia socioeconómica y ubica- ción, para cada una de las ciénagas. Para ello se establecieron los siguientes porcentajes: la extensión de cada ciénaga equivale a un $20 \%$ de importancia, la ubicación a $35 \%$ y las actividades socioeconómicas a un $45 \%$. De esta manera se tiene que:

$$
\text { Grado de vulnerabilidad }=\mathrm{GD}
$$

$\mathrm{GD}=($ Valor de calificación de extensión* 0.20$)+($ Valor de calificación de ubicación * 0.35) + (Valor de calificación de importancia socioeconómica * 0.45)

Como resultado de estos cálculos se obtienen las ciénagas priorizadas de acuerdo con el máximo valor obtenido de grado de vulnerabilidad. Finalmente se determinaron además los rangos de vulnerabilidad para cada una de las ciénagas dependiendo del valor obtenido (Tabla 3 ).

\section{RESULTADOSY DISCUSIÓN}

Inventario, ubicación y extensión de ciénagas. Unido al sistema hidrológico del municipio del Medio Atrato se encuentran 47 ciénagas reportadas cartográficamente y reconocidas en el trabajo de campo en el área de estudio. En la Tabla

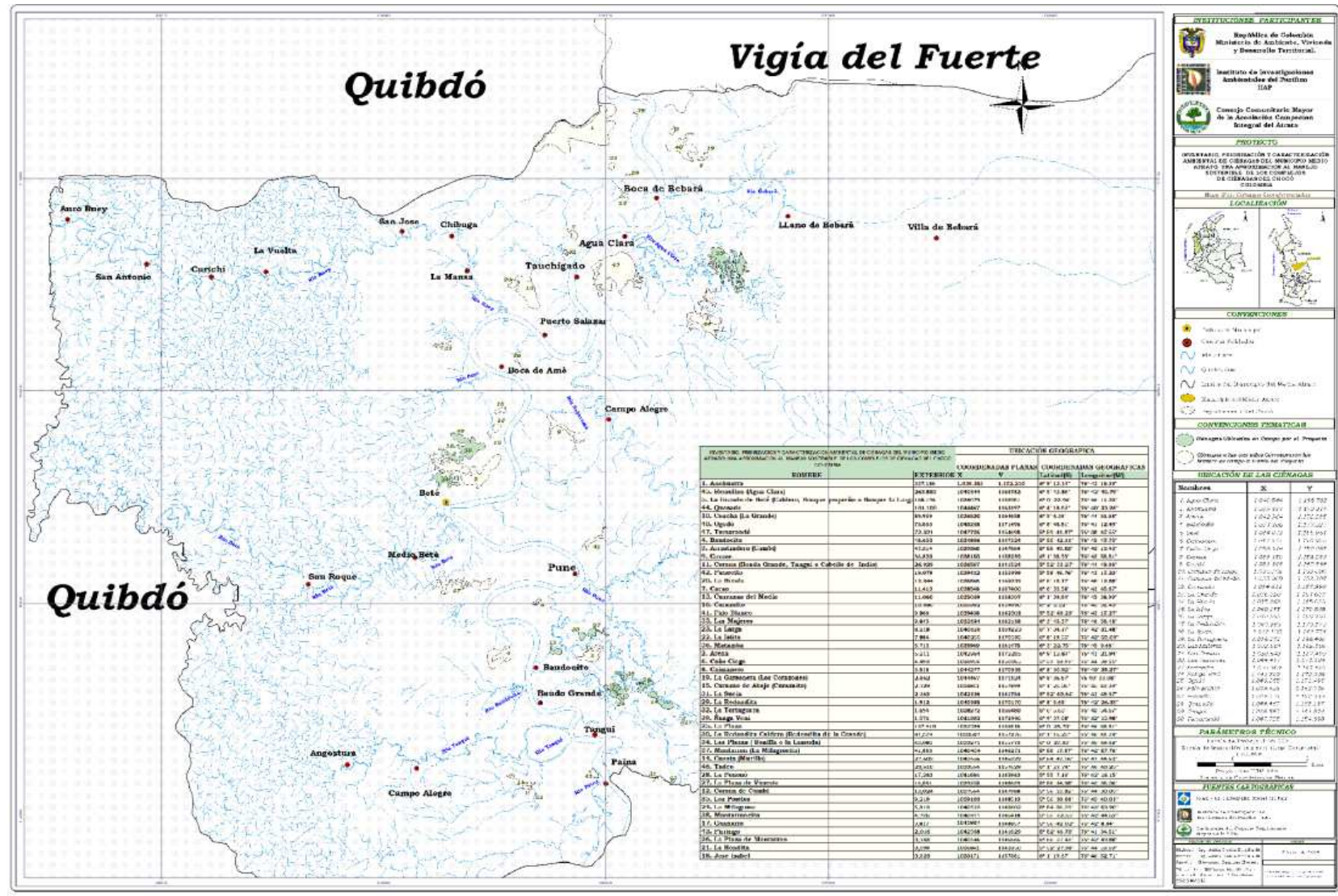

Figura 1. Inventario de ciénagas 
Tabla 2 Rangos de calificación del uso de las ciénagas

\begin{tabular}{|c|c|}
\hline Usos & lor de calificación \\
\hline Pesca & 1 \\
\hline Minería & 1 \\
\hline Extracción forestal & 1 \\
\hline Pesca + minería & 3 \\
\hline Pesca + extracción forestal & 3 \\
\hline Minería + extracción forestal & 3 \\
\hline Minería + extracción forestal + pesca & 5 \\
\hline \multicolumn{2}{|c|}{$\begin{array}{c}\text { Tabla } 3 \\
\text { Rangos de calificación del grado de vulnerabili- } \\
\text { dad de las ciénagas }\end{array}$} \\
\hline Grado de vulnerabilidad & Rango \\
\hline Alto & $4-5$ \\
\hline Medio & $2.8-3.9$ \\
\hline Bajo & $0-2.7$ \\
\hline
\end{tabular}

4 se muestra la relación de dichas ciénagas, con una descripción breve de la ubicación o ruta de acceso, y datos de extensión y georeferenciación (Figura 1).

Actividades socioeconómicas en las ciénagas. Las comunidades negras ubicadas en toda la cuenca del río Atrato organizadas en consejos comunitarios han distribuido geográficamente la región en 9 zonas delimitadas no sólo a nivel territorial sino también en los aspectos sociales, culturales y económicos. Al municipio del Medio Atrato hacen parte las zonas $1,3,4,5$ y 6 , de las cuales la zona 1 no presenta ciénagas ni explota los recursos en áreas aledañas y la zona 6, a pesar de tener ciénagas en territorio chocoano on explotadas por la comunidad de Palo Blanco (Antioquia), no serán tenidas en cuenta como zonas de estudio. En las zonas 3 y 5 se practica de manera simultánea la minería, la extracción de madera y la pesca y en la zona 4 solamente el corte de madera y la pesca. En la Tabla 5 se muestran las actividades socioeconómicas o la importancia sociocultural por ciénaga y el respectivo grado de vulnerabilidad obtenido.

Ponderación y priorización. Como resultado de los talleres de socialización realizados con la comunidad de la zona de estudio y las observaciones en campo del equipo técnico se pudo determinar que las ciénagas pertenecientes al municipio del Medio Atrato juegan un papel muy importante en la vida de sus habitantes, porque en estos ecosistemas se desarrolla gran parte de las actividades productivas para su subsisten- cia, teniendo en cuenta que estas producen y consumen por tradición los productos generados a partir de los recursos de los mismos. Esto les ha permitido sobrevivir y proteger parte de estos recursos.

Sin embargo en las últimas décadas las ciénagas de la zona están sufriendo un deterioro acelerado debido a la sobreexplotación de sus recursos, todo esto a causa de obtener beneficios económicos de manera más fácil y rápida, poniendo en riesgo el equilibrio y provocando la pérdida de biodiversidad.

Las comunidades asentadas en esta región de acuerdo con sus criterios organizativos han dividido toda la cuenca del río Atrato en varias zonas, siendo las zonas 3, 4 y 5 las correspondientes al municipio de Medio Atrato, donde se encuentra un complejo de aproximadamente 78 ciénagas, que en la actualidad se desarrollan actividades económicas como la extracción de madera para comercialización, seguida de la minería, la pesca y en menor grado la agricultura. Estas actividades productivas a su vez, están distribuidas en las zonas mencionadas, presentándose en la zona 4 la extracción de madera y agricultura en menor escala y en las zonas 3 y 5 se realizan de manera simultánea la extracción de madera y la minería, de ahí los resultados obtenidos en la priorización. La comunidad junto con el equipo técnico teniendo en cuenta los criterios de selección, determinaron que la ciénaga que requiere la caracterización es la Grande de Beté (Tablas 6, 7 y 8).

La metodología construida para seleccionar una sola ciénaga, donde se pudiera realizar una caracterización ambiental amplia en diversos componentes, se basó en las teorías existentes sobre alternativas estratégicas para la toma de decisiones en la administración de empresas, análisis multicriterio y ponderación y determinación de criterios, que surgen como una respuesta a una de las etapas más importantes de la resolución de cualquier problema de decisión multicriterio, que es la identificación y ponderación de los mismos, según Nieto (2001), que además expresa que determinar la importancia relativa de los criterios no es una labor sencilla ni fiable debido a la subjetividad propia de dicha valoración. Los métodos de ponderación clásicos desarrollados requieren datos de partida precisos, siendo imposible en muchos casos, definir datos de esta naturaleza.

En este sentido Delgado et al. (2008), sostienen que es bastante conveniente en la decisión multicriterio que unos criterios tengan para el decisor más relevancias que otros. Por circunstancias muy diversas, entre las que lógicamente están sus preferencias personales, el decisor puede considerar más o menos importante a un criterio que a los restantes. Se denominan pesos (o ponderaciones) a estas medidas de la importancia relativa que los criterios tienen para el decisor. A su vez expresan que es deseable que los criterios sean evaluados sobre escalas comparables en tipo, rango de extensión, unidad de medida, eventual posición del cero, disper- 


\section{Bioetnia Volumen 6 No 2 (julio-diciembre), 2009}

Tabla 4

Ubicación geográfica de las ciénagas

\begin{tabular}{|c|c|c|c|c|c|c|}
\hline Ciénagas & Ubicación & Área (ha) & $\mathbf{X}$ & $\mathbf{Y}$ & Latitud (N) & Longitud (W) \\
\hline Anchuarra & $\begin{array}{l}\text { Se encuentra ubicada a la margen } \\
\text { izquierda del río Atrato, conectándose } \\
\text { con el mismo a través de un caño del } \\
\text { mismo nombre. }\end{array}$ & 337.186 & 1.039 .353 & 1.172 .210 & 6ㅇ' $12.14 "$ & 76우 $43^{\prime} 19.39 "$ \\
\hline $\begin{array}{l}\text { Agua Clara, } \\
\text { Remolino }\end{array}$ & $\begin{array}{l}\text { Se conecta a través de un caño a la } \\
\text { quebrada del mismo nombre. }\end{array}$ & 263.850 & 1040544 & 1165782 & $605^{\prime} 42.86 "$ & $76 \circ 42^{\prime} 40.79^{\prime \prime}$ \\
\hline $\begin{array}{l}\text { La Grande } \\
\text { de Beté }\end{array}$ & $\begin{array}{l}\text { Está localizada a la margen izquierda del } \\
\text { río Atrato aproximadamente a un } 1 \mathrm{~km} \text {. }\end{array}$ & 168.194 & 1034079 & 1155951 & 6음 0 '22.96" & $76 \circ 46^{\prime} 11.23 "$ \\
\hline Quesada & $\begin{array}{l}\text { Se localiza a la margen derecha sobre el } \\
\text { río Atrato con el que se conecta por los } \\
\text { caños de Tauchigadó y Agua Clara. }\end{array}$ & 101.100 & 1044467 & 1163197 & 6은 '18.62" & $76 \div 40^{\prime} 33.28 "$ \\
\hline $\begin{array}{l}\text { La Concha, } \\
\text { La Grande }\end{array}$ & $\begin{array}{l}\text { Se hace conexión con esta ciénaga a } \\
\text { través de la quebrada Buey Viejo. }\end{array}$ & 89.959 & 1036520 & 1164658 & $6^{\circ} 5^{\prime} 6.36^{\prime \prime}$ & $76^{\circ} 44^{\prime} 51.68 "$ \\
\hline Ogodó & $\begin{array}{l}\text { Se conecta por la quebrada Ogodó a } \\
\text { través de un caño del mismo nombre, } \\
\text { que se localiza en la margen derecha del } \\
\text { río Atrato. }\end{array}$ & 73.553 & 1043255 & 1171496 & $608^{\prime} 48.81^{\prime \prime}$ & $76 \circ 41^{\prime} 12.49^{\prime \prime}$ \\
\hline Tumarado & $\begin{array}{l}\text { Está conectada directamente con la } \\
\text { quebrada Tumaradó. }\end{array}$ & 72.321 & 1047725 & 1154698 & 5은 $59^{\prime} 41.87 "$ & $76^{\circ} 38^{\prime} 47.55^{\prime \prime}$ \\
\hline Baudocito & $\begin{array}{l}\text { Ubicada en la margen izquierda del río } \\
\text { Atrato y se conecta a través del caño } \\
\text { Baudocito }\end{array}$ & 48.650 & 1034806 & 1147324 & $5^{\circ} 55^{\prime} 42.11^{\prime \prime}$ & $76045^{\prime} 47.75^{\prime \prime}$ \\
\hline $\begin{array}{l}\text { Cumbí, } \\
\text { Arrastradero }\end{array}$ & $\begin{array}{l}\text { Se ubica en la margen derecha del río } \\
\text { Atrato y se conecta por el caño Ciego. }\end{array}$ & 47.314 & 1039368 & 1147564 & 5o 55' 49.83" & $76^{\circ} 43^{\prime} 19.43^{\prime \prime}$ \\
\hline Cavase & $\begin{array}{l}\text { Conectada con el río Atrato a través del } \\
\text { caño Casave, el cual se encuentra a la }\end{array}$ & 36.823 & 1038150 & 1158289 & 6o 1' 38.99" & 76o 43' 58.81" \\
\hline & margen izquierda del río Atrato. & 26.929 & 1036587 & 1141524 & 5o $52^{\prime} 33.27^{\prime \prime}$ & $76044^{\prime} 49.96 "$ \\
\hline Tanguíi ${ }^{2}$ & $\begin{array}{l}\text { Está conectada directamente con el río } \\
\text { Tanguí. }\end{array}$ & & & & & \\
\hline Punecito & $\begin{array}{l}\text { Está conectada con el río Pune a través } \\
\text { del caño Punecito. }\end{array}$ & 19.079 & 1039432 & 1152999 & $5058^{\prime} 46.76^{\prime \prime}$ & $76043^{\prime} 17.23 "$ \\
\hline La Honda & $\begin{array}{l}\text { Pertenece a la quebrada Buey Viejo la } \\
\text { cual hace conexión con la ciénaga La Grande. }\end{array}$ & 12.044 & 1035868 & 1165039 & $605^{\prime} 18.77 "$ & $76045^{\prime} 12.88^{\prime \prime}$ \\
\hline Los Cacaos & $\begin{array}{l}\text { Esta quebrada es afluente de la quebrada } \\
\text { Muerteros. }\end{array}$ & 11.410 & 1038548 & 1167400 & 6 6' $35.58^{\prime \prime}$ & $76043^{\prime} 45.67 "$ \\
\hline $\begin{array}{l}\text { Curazao } \\
\text { de Medio }\end{array}$ & $\begin{array}{l}\text { Se comunica con el río Atrato a través del } \\
\text { caño Inglés. Está localizada a la margen } \\
\text { derecha del río Atrato y se accede por el } \\
\text { río Ame a través de un caño del mismo } \\
\text { nombre. }\end{array}$ & 11.060 & 1035069 & 1158307 & 6ㅇ 1' 39.64" & $76045^{\prime} 38.99 "$ \\
\hline Curazaito & $\begin{array}{l}\text { Está localizada a margen derecha del río } \\
\text { Atrato y se accede por el río Ame a través } \\
\text { de un caño del mismo nombre. }\end{array}$ & 10.086 & 1035302 & 1159090 & 6으 2' 5.12" & $76045^{\prime} 31.40 "$ \\
\hline Paloblanco & $\begin{array}{l}\text { Está conectada directamente con el río } \\
\text { Atrato sobre la margen izquierda. }\end{array}$ & 9.866 & 1039438 & 1142018 & 5o $52^{\prime} 49.29^{\prime \prime}$ & $76043^{\prime} 17.27 "$ \\
\hline Las Mujeres & $\begin{array}{l}\text { Ubicada a la margen izquierda del río Atrato } \\
\text { y está conectada con el mismo a través } \\
\text { del caño Amorcito. }\end{array}$ & 9.845 & 1032684 & 1162168 & 6o 3' 45.37" & $76046^{\prime} 56.48^{\prime \prime}$ \\
\hline La Larga & $\begin{array}{l}\text { Se conecta con el río Atrato a través del } \\
\text { caño La Larga. }\end{array}$ & 8.218 & 1040828 & 1169220 & 6 ' $34.77^{\prime \prime}$ & $76^{\circ} 42^{\prime} 31.48 "$ \\
\hline La Islita & $\begin{array}{l}\text { Se localiza a la margen izquierda del río } \\
\text { Atrato y se conecta a través del caño Inglés. }\end{array}$ & 7.884 & 1040255 & 1170595 & 6우 8' 19.55" & 76임 $50.09 "$ \\
\hline Matamba & $\begin{array}{l}\text { Se ubica en la margen derecha del río Atrato } \\
\text { a través de un caño del mismo nombre. }\end{array}$ & 5.713 & 1035969 & 1161475 & 6ㅇ $3^{\prime} 22.75^{\prime \prime}$ & 76우 45' 9.66" \\
\hline Arena & $\begin{array}{l}\text { Conectada directamente con la Quebrada } \\
\text { Ogodó a través del caño del mismo nombre, } \\
\text { ubicada en la margen derecha del río Atrato. }\end{array}$ & 5.211 & 1042964 & 1172235 & 6ㅇ 9' 12.87" & $76 \div 41^{\prime} 21.94 "$ \\
\hline
\end{tabular}

1. Caldero, bosque pequeño o bosque largo $\quad$ 2. Corona, Cabello de Indio, Honda Grande 
Herramienta metodológica para priorizar ecosistemas estratégicos. G Ramírez et al.

Tabla 4

Ubicación geográfica de las ciénagas (continuación)

\begin{tabular}{|c|c|c|c|c|c|c|}
\hline Ciénagas & Ubicación & Área (ha) & $\mathbf{x}$ & $\mathbf{Y}$ & Latitud (N) & Longitud (W) \\
\hline Caño Ciego & $\begin{array}{l}\text { Está localizada a la margen derecha del río } \\
\text { Atrato y se conecta a través del caño Ciego. }\end{array}$ & 4.490 & 1036914 & 1150055 & $5^{\circ} 57^{\prime} 10.97^{\prime \prime}$ & $76^{\circ} 44^{\prime} 39.16^{\prime \prime}$ \\
\hline Caimanero & $\begin{array}{l}\text { A esta ciénaga se accede por la quebrada } \\
\text { Ogodó y el caño del mismo nombre. }\end{array}$ & 3.518 & 1044277 & 1170935 & 6o 8' 30.52" & $76 \cong 40^{\prime} 39.27 "$ \\
\hline $\begin{array}{l}\text { Los } \\
\text { Garzonera }\end{array}$ & $\begin{array}{l}\text { Está localizada a la margen derecha del río } \\
\text { Atrato y se accede por la quebrada Ogodó a } \\
\text { través del caño Ogodó. }\end{array}$ & 2.862 & 1044467 & 1171124 & $6^{\circ} 8^{\prime} 36.67^{\prime \prime}$ & $7640^{\prime} 33.08^{\prime \prime}$ \\
\hline $\begin{array}{l}\text { Curazao de } \\
\text { Abajo }\end{array}$ & $\begin{array}{l}\text { Está localizada a la margen derecha del río } \\
\text { Atrato y se accede por el río Ame a través de } \\
\text { un caño del mismo nombre. }\end{array}$ & 2.729 & 1034811 & 1157899 & 6o 1' 26.36" & $76^{\circ} \quad 45^{\prime} 47.39^{\prime \prime}$ \\
\hline La Sucia & $\begin{array}{l}\text { Se ubica sobre la margen derecha del río } \\
\text { Atrato y se conecta con él a través del caño } \\
\text { La Sucia. }\end{array}$ & 2.363 & 1042136 & 1141754 & $5^{\circ} 52^{\prime} 40.64^{\prime \prime}$ & $76 \circ 41^{\prime} 49.57^{\prime \prime}$ \\
\hline La Redondita & $\begin{array}{l}\text { Está localizada a la margen izquierda del río } \\
\text { Atrato y se accede por el caño Inglés. }\end{array}$ & 1.912 & 1040985 & 1170170 & 6을 5.69" & $76^{\circ} 42^{\prime} 26.35^{\prime \prime}$ \\
\hline La Tortuguera & $\begin{array}{l}\text { Conectada directamente con la quebrada } \\
\text { Muerteros que a su vez es afluente del río } \\
\text { Atrato. }\end{array}$ & 1.694 & 1038272 & 1166480 & 6ㅇ' 6. 5.63" & 76 $43^{\prime} 54.67 "$ \\
\hline Ñanga Vení & $\begin{array}{l}\text { Está conectada directamente con el río Atrato, } \\
\text { a una distancia aproximada de } 500 \text { m sobre la } \\
\text { margen izquierda. }\end{array}$ & 1.574 & 1041302 & 1172996 & 6o 9' 37.68" & $76^{\circ} 42^{\prime} 15.98^{\prime \prime}$ \\
\hline La Plaza & $\begin{array}{l}\text { Se accede por el río Agua Clara, pasando por } \\
\text { la ciénaga la Quesada. }\end{array}$ & 137.410 & 1032984 & 1155815 & 6음 0 ' 28.70" & $76^{\circ} 46^{\prime} 58.81^{\prime \prime}$ \\
\hline $\begin{array}{l}\text { La Redondita } \\
\text { de Caldero }\end{array}$ & $\begin{array}{l}\text { Se accede por el río Atrato pasando por } \\
\text { ciénaga la Grande de Beté a través del caño } \\
\text { Torrobón. }\end{array}$ & 81.379 & 1033507 & 1157276 & 6우 1' 16.25" & $76046^{\prime} 41.78 "$ \\
\hline Las Plazas ${ }^{4}$ & $\begin{array}{l}\text { Se accede por el río Atrato pasando por } \\
\text { ciénaga la Grande de Beté a través del caño } \\
\text { Torrobón. }\end{array}$ & 43.081 & 1033271 & 1155773 & 6o $0^{\prime} 27.33^{\prime \prime}$ & $76^{\circ} 46^{\prime} 49.48^{\prime \prime}$ \\
\hline Montarrón 5 & $\begin{array}{l}\text { Se encuentra conectada directamente con el } \\
\text { río Puné, pasando por las ciénagas, Pononó, } \\
\text { Montarroncito y la Plaza de Montarron. }\end{array}$ & 41.883 & 1040404 & 1146271 & $5^{\circ} 55^{\prime} 17.87^{\prime \prime}$ & $76^{\circ} 42^{\prime} 57.76^{\prime \prime}$ \\
\hline $\begin{array}{l}\text { Cuesta } \\
\text { (Murillo) }\end{array}$ & $\begin{array}{l}\text { Esta conectada directamente con el río Puné a } \\
\text { través de la ciénaga Pononó. }\end{array}$ & 27.603 & 1042655 & 1145329 & $5^{\circ} 54^{\prime} 47.16^{\prime \prime}$ & $76 \circ 41^{\prime} 44.60^{\prime \prime}$ \\
\hline Tadeo & $\begin{array}{l}\text { A esta ciénaga se accede a través de la } \\
\text { ciénaga la Redondita de Caldero por el caño } \\
\text { Torrobón. }\end{array}$ & 23.610 & 1033554 & 1157629 & 6ㅇ' 1' 27.74" & $76^{\circ} 46^{\prime} 40.25^{\prime \prime}$ \\
\hline La Pononó & $\begin{array}{l}\text { Esta conectada directamente con el río Puné } \\
\text { pasando por la ciénaga Cuesta. }\end{array}$ & 17.243 & 1041684 & 1145943 & $5^{\circ} 55^{\prime} 7.16^{\prime \prime}$ & $76^{\circ} 42^{\prime} 16.15^{\prime \prime}$ \\
\hline $\begin{array}{l}\text { La Plaza de } \\
\text { Vicente }\end{array}$ & $\begin{array}{l}\text { Se encuentra conectada directamente al río } \\
\text { Puné, a través de un caño corto. }\end{array}$ & 16.581 & 1039255 & 1148639 & $5^{\circ} 56^{\prime} 34.98 "$ & $76^{\circ} 43^{\prime} 35.06 "$ \\
\hline $\begin{array}{l}\text { Corona de } \\
\text { Cumbí }\end{array}$ & $\begin{array}{l}\text { Se ubica sobre la margen izquierda del río } \\
\text { Atrato y se conecta en forma directa. }\end{array}$ & 13.024 & 1037564 & 1147988 & $5^{\circ} 56^{\prime} 13.82 "$ & $76 \circ 44^{\prime} 30.05^{\prime \prime}$ \\
\hline Los Positos & $\begin{array}{l}\text { Esta ubicada en la margen derecha del río } \\
\text { Puné, conectándose de forma directa a éste. }\end{array}$ & 9,219 & 1039103 & 1148513 & $5^{\circ} 56^{\prime} 30.88^{\prime \prime}$ & 76 $43^{\prime} 40.01 "$ \\
\hline La Milagrosa & $\begin{array}{l}\text { Se accede a ella ingresando por el río Puné a } \\
\text { través de la ciénaga Montarron. }\end{array}$ & 5.310 & 1040523 & 1145602 & $5^{\circ} 54^{\prime} 56.09 "$ & $76^{\circ} 42^{\prime} 53.90 "$ \\
\hline Montarroncito & $\begin{array}{l}\text { Se encuentra directamente conectada con el } \\
\text { río Puné sobre su margen izquierda. }\end{array}$ & 4.726 & 1040811 & 1146418 & 5o 55' 22.65" & $76^{\circ} 42^{\prime} 44.52^{\prime \prime}$ \\
\hline Guanarro & $\begin{array}{l}\text { Se conecta con el río Puné pasando por la } \\
\text { ciénaga Cuesta. }\end{array}$ & 3.817 & 1941907 & 1148857 & 5 $56^{\prime} 42.02 "$ & $76^{\circ} 4^{\prime \prime} 8.84 "$ \\
\hline Pirringo & $\begin{array}{l}\text { Conectada con el río Paina a través de la ciénaga } \\
\text { la Sucia.Se accede a ella por la ciénaga }\end{array}$ & 2.016 & 1042968 & 1141629 & $5^{\circ} 52^{\prime} 46.70^{\prime \prime}$ & $76 \circ 41^{\prime} 34.51 "$ \\
\hline $\begin{array}{l}\text { La Plaza de } \\
\text { Montarron }\end{array}$ & $\begin{array}{l}\text { Montarroncito que a su vez se encuentra } \\
\text { conectada con el río Puné. }\end{array}$ & 0.783 & 1040646 & 1146565 & $5^{\circ} 55^{\prime} 27.43^{\prime \prime}$ & $76 \div 42^{\prime} 49.88^{\prime \prime}$ \\
\hline La Hondita & $\begin{array}{l}\text { Se encuentra conectada a la ciénaga la Corona, } \\
\text { ingresando por el río Tanguí. }\end{array}$ & 0.399 & 1036841 & 1141050 & 5 52 ' 27.89" & 76 $44^{\prime} 53.69 "$ \\
\hline
\end{tabular}

3. Redondita de la Grande 4.Bonilla, Lamuda 5.La Milagrosita 
Bioetnia Volumen 6 No 2 (julio-diciembre), 2009

Tabla 4

Ubicación geográfica de las ciénagas (continuación)

\begin{tabular}{llccccc}
\hline Ciénagas & Ubicación & Área (ha) & $\mathbf{X}$ & $\mathbf{Y}$ & Latitud (N) & Longitud (W) \\
\hline José Isabel & $\begin{array}{l}\text { Se ingresa a través de la ciénaga la } \\
\text { Redondita de Caldero. }\end{array}$ & 0.323 & 1033171 & 1157381 & $6^{\circ}$ 41' 19.67" & 760 46' 52.71" \\
\hline
\end{tabular}

sión para obtener resultados confiables y que se puedan comparar y analizar con facilidad. Esta situación se cumplió dentro de la metodología diseñada para seleccionar una ciénaga dentro de tantas localizadas en el mismo complejo del área de estudio, evaluando para cada una los mismo criterios, con sus respectivos pesos o grados de importancia, asignados de tal manera que resultara el ecosistema como mejor estado de conservación y más urgencia de estudio.

\section{CONCLUSIONES}

El complejo de ciénagas del municipio de Medio Atrato, está constituido por 47 cuerpos de agua, los cuales se encuentran relativamente bien conservados, en tanto que aún se registra una gran riqueza en cuanto a recursos bióticos y abióticos; sin embargo, se avizoran algunos factores produc- tivos como la pesca, la minería y la extracción maderera que podrían atentar contra la sostenibilidad de dichos recursos en el mediano y largo plazo.

De otro lado, es importante anotar que la herramienta metodológica construida y aplicada en el ejercicio de campo, funciona adecuadamente; sin embargo, se requieren ejercicios complementarios que permitan ir ajustando la herramienta y de esta forma no sólo lograr la priorización de ecosistemas cenagosos, sino también otros tipos de ecosistemas estratégicos.

\section{LITERATURA CITADA}

Nieto, A. 2001. Contribución a la ponderación de criterios en problemas de toma de decisión en proyectos en ambientes difusos. Tesis de Grado. Universidad Politécnica de Valencia, España.

Delgado, F., García, J., Abreu, R., R. Orozco. 2008. Los métodos de ponderación y sus problemas. Monografía. Universidad Central de Las Villas. Santa Clara, Cuba. 
Herramienta metodológica para priorizar ecosistemas estratégicos. G Ramírez et al.

Tabla 5

Actividades socioeconómicas y grado de vulnerabilidad por ciénaga

\begin{tabular}{|c|c|c|c|c|c|c|c|}
\hline \multirow[t]{2}{*}{$\mathbf{N}$} & \multirow{2}{*}{$\begin{array}{l}\text { Nombre de la } \\
\text { ciénaga }\end{array}$} & \multicolumn{3}{|c|}{ Usos } & \multicolumn{3}{|c|}{ Grado de vulnerabilidad } \\
\hline & & Pesca & Mineria & Corte de madera & $\mathbf{A}$ & M & B \\
\hline 1 & Anchuarra & & $x$ & & & & $x$ \\
\hline 2 & La Quesada & & $\mathrm{x}$ & $\mathrm{x}$ & & $\mathrm{x}$ & \\
\hline 3 & Arena & $x$ & $\mathrm{x}$ & $x$ & $x$ & & \\
\hline 4 & Baudocito & $x$ & & $\mathrm{x}$ & & $\mathrm{x}$ & \\
\hline 5 & Caimanero & $\mathrm{x}$ & & $\mathrm{x}$ & & & $x$ \\
\hline 6 & Caño Ciego & $x$ & & $x$ & & $x$ & \\
\hline 7 & Casave & $\mathrm{x}$ & $\mathrm{x}$ & $\mathrm{X}$ & $\mathrm{X}$ & & \\
\hline 8 & Cumbi & $x$ & $x$ & $x$ & & $x$ & \\
\hline 9 & Ogodo & $x$ & $x$ & $x$ & $x$ & & \\
\hline 10 & Curazao de Abajo & $x$ & & $\mathrm{x}$ & & & $\mathrm{x}$ \\
\hline 11 & La Islita & $x$ & $x$ & $x$ & & $x$ & \\
\hline 12 & Los Garzonera & $\mathrm{x}$ & & $\mathrm{x}$ & & & $\mathrm{x}$ \\
\hline 13 & Curazaito & $\mathrm{x}$ & & $\mathrm{x}$ & & & $x$ \\
\hline 14 & La Redondita & $x$ & $x$ & $x$ & $x$ & & \\
\hline 15 & Ñanga veni & $\mathrm{x}$ & $\mathrm{x}$ & $\mathrm{x}$ & $\mathrm{X}$ & & \\
\hline 16 & Curazao del Medio & $x$ & & $x$ & & & $x$ \\
\hline 17 & Palo Blanco & $\mathrm{x}$ & $\mathrm{x}$ & $x$ & $\mathrm{X}$ & & \\
\hline 18 & La Sucia & $x$ & $x$ & $x$ & $x$ & & \\
\hline 19 & Tumarado & $\mathrm{x}$ & & $\mathrm{x}$ & & $\mathrm{X}$ & \\
\hline 20 & Tangui & $x$ & & $x$ & & $x$ & \\
\hline 21 & Las Mujeres & $\mathrm{x}$ & & $x$ & & $x$ & \\
\hline 22 & Matamba & $x$ & & $x$ & & $\mathrm{X}$ & \\
\hline 23 & La Honda & $x$ & & $\mathrm{x}$ & & & $\mathrm{x}$ \\
\hline 24 & Los Cacaos & $x$ & $x$ & $x$ & & $\mathrm{x}$ & \\
\hline 25 & La Grande de Beté & $\mathrm{X}$ & $\mathrm{X}$ & $\mathrm{X}$ & $\mathrm{x}$ & & \\
\hline 26 & La Tortuguera & $x$ & $x$ & $x$ & $x$ & & \\
\hline 27 & La Larga & $x$ & $x$ & $x$ & $x$ & & \\
\hline 28 & Agua Clara & $\mathrm{x}$ & & $\mathrm{x}$ & & $\mathrm{x}$ & \\
\hline 29 & Pirringo & $x$ & $x$ & $x$ & $x$ & & \\
\hline 30 & La Hondita & $\mathrm{x}$ & $\mathrm{x}$ & $\mathrm{X}$ & $\mathrm{X}$ & & \\
\hline 31 & Los Positos & $\mathrm{x}$ & & $\mathrm{x}$ & & $\mathrm{x}$ & \\
\hline 32 & Cuesta & $\mathrm{x}$ & & $\mathrm{x}$ & & $\mathrm{x}$ & \\
\hline 33 & La Pononó & $x$ & & $x$ & & $x$ & \\
\hline 34 & La Plaza de Montarrón & $\mathrm{x}$ & & $\mathrm{X}$ & & $\mathrm{x}$ & \\
\hline 35 & La Milagrosa & $\mathrm{x}$ & & $\mathrm{x}$ & & $\mathrm{x}$ & \\
\hline 36 & Montarroncito & $\mathrm{x}$ & & $\mathrm{x}$ & & $\mathrm{x}$ & \\
\hline 37 & Corona de Cumbí & $x$ & & $x$ & & $x$ & \\
\hline 38 & La Plaza de Vicente & $\mathrm{x}$ & & $\mathrm{X}$ & & $\mathrm{x}$ & \\
\hline 39 & Tadeo & $\mathrm{x}$ & & $x$ & & $\mathrm{x}$ & \\
\hline 40 & La Redondita de la Grand & hde $X$ & & $\mathrm{x}$ & & $\mathrm{x}$ & \\
\hline 41 & José Isabel & $x$ & & $x$ & & $x$ & \\
\hline 42 & La Plaza & $x$ & & $x$ & & $x$ & \\
\hline 43 & La Concha & $\mathrm{X}$ & & $X$ & & $\mathrm{X}$ & \\
\hline 44 & Guanarro & $x$ & & $x$ & & $x$ & \\
\hline 45 & Punecito & $\mathrm{x}$ & $\mathrm{X}$ & $\mathrm{X}$ & $\mathrm{X}$ & & \\
\hline 46 & Las Plazas (Bonilla, Lamu & nuda) & $\mathrm{x}$ & & $\mathrm{X}$ & & $\mathrm{x}$ \\
\hline 47 & Montarrón & $x$ & & $x$ & & $x$ & \\
\hline
\end{tabular}


Bioetnia Volumen 6 No 2 (julio-diciembre), 2009

Tabla 6

Ponderación de los criterios de las ciénagas de la zona 3

\begin{tabular}{lcccc}
\hline Ciénagas & \multicolumn{3}{c}{ Criterios de priorización } & \\
\cline { 2 - 3 } & Extensión & Ubicación & $\begin{array}{c}\text { Importancia } \\
\text { socioeconómica }\end{array}$ & Grado vulnerabilidad \\
\hline Casave & 1 & 5 & 5 & 4,2 \\
Cumbi (Arrastradero) & 1 & 3 & 5 & 3,5 \\
Ñanga Veni & 1 & 5 & 5 & 4,2 \\
La Sucia & 1 & 5 & 5 & 4,2 \\
Los Cacaos & 1 & 3 & 5 & 3,5 \\
La Tortuguera & 1 & 5 & 5 & 4,2 \\
Pirringo & 1 & 3 & 5 & 3,5 \\
Tangui (Corona, Cabello de Indio) & 1 & 3 & 5 & 3,5 \\
La Hondita & 1 & 3 & 5 & 3,5 \\
\hline
\end{tabular}

Tabla 7

Ponderación de los criterios de las ciénagas de la zona 4

\begin{tabular}{|c|c|c|c|}
\hline Ciénagas & & erios de pri & ción \\
\hline & Extensión & Ubicación & $\begin{array}{c}\text { Importancia } \\
\text { socioeconómica }\end{array}$ \\
\hline
\end{tabular}

\begin{tabular}{|c|c|c|c|c|}
\hline La Grande de Beté & 2 & 5 & 5 & $4,4 *$ \\
\hline Baudocito & 1 & 5 & 3 & 3,3 \\
\hline Caimanero & 1 & 3 & 3 & 2,6 \\
\hline Caño Ciego & 1 & 5 & 3 & 3,3 \\
\hline Curazao de Abajo & 1 & 3 & 3 & 2,6 \\
\hline La Garzonera & 1 & 3 & 3 & 2,6 \\
\hline Curazaito & 1 & 3 & 3 & 2,6 \\
\hline Curazao del Medio & 1 & 3 & 3 & 2,6 \\
\hline Punecito & 1 & 3 & 3 & 2,6 \\
\hline Tumarado & 1 & 5 & 3 & 3,3 \\
\hline Las Mujeres & 1 & 5 & 3 & 3,3 \\
\hline Matamba & 1 & 5 & 3 & 3,3 \\
\hline La Honda & 1 & 3 & 3 & 2,6 \\
\hline Guanarro & 1 & 3 & 3 & 3,7 \\
\hline Los Positos & 1 & 5 & 3 & 2,6 \\
\hline Cuesta & 1 & 3 & 3 & 3,3 \\
\hline La Pononó & 1 & 3 & 3 & 2,6 \\
\hline La Plaza de Montarrón & 1 & 3 & 3 & 2,6 \\
\hline Montarrón (La Milagrosita) & 1 & 3 & 5 & 2,6 \\
\hline La Milagrosa & 1 & 3 & 3 & 3,5 \\
\hline Montarroncito & 1 & 3 & 5 & 2,6 \\
\hline Corona de Cumbí & 1 & 5 & 5 & 3,5 \\
\hline La Plaza de Vicente & 1 & 5 & 5 & 4,2 \\
\hline La Redondita de la Grande o de Caldero & 1 & 3 & 3 & 4,2 \\
\hline José Isabel & 1 & 3 & 3 & 2,6 \\
\hline Las Plazas (Lamuda, Bonilla) & 1 & 3 & 3 & 2,6 \\
\hline La Concha & 1 & 5 & 3 & 2,6 \\
\hline
\end{tabular}

* La ciénaga priorizada de la zona 4 es La Grande de Beté 
Herramienta metodológica para priorizar ecosistemas estratégicos. G Ramírez et al.

Tabla 8

Ponderación de los criterios de las ciénagas de la zona 5

\begin{tabular}{lcccc}
\hline Ciénagas & \multicolumn{2}{c}{ Criterios de priorización } & \\
\cline { 2 - 3 } & Extensión & Ubicación & $\begin{array}{c}\text { Importancia } \\
\text { socioeconómica }\end{array}$ & Grado vulnerabilidad \\
\hline Achuarra & 5 & 5 & 1 & 3,2 \\
Quesada & 2 & 5 & 3 & 3,5 \\
Arena & 1 & 5 & 5 & 4,2 \\
Agua Clara & 4 & 5 & 5 & 2,4 \\
La Larga & 1 & 5 & 1 & 4,2 \\
Palo Blanco & 1 & 5 & 5 & 4,2 \\
Ogodo & 1 & 5 & 5 & 4,2 \\
La Islita & 1 & 3 & 5 & 3,5 \\
La Redondita & 1 & 5 & 5 & 4,2 \\
La Plaza & 2 & 3 & 3 & 3,7 \\
\hline
\end{tabular}

